\title{
La colaboración científica y la internacionalización de la ciencia mexicana de 1980 a 2004
}

\author{
María Elena Luna-Morales *
}

Artículo recibido:

15 de marzo de 2012.

Artículo aceptado:

11 de mayo de 2012.

\section{RESUMEN}

Este trabajo presenta un análisis de las publicaciones registradas en las bases de datos Science Citation Index (SCI) en el periodo 1980-2004, y Social Science Citation Index (SSCI) de 1997-2004, para determinar el nivel de internacionalización que sostiene la ciencia mexicana, tomando como aspecto básico de estudio la colaboración científica establecida con otras instituciones, países y disciplinas científicas. El trabajo se apoya en el método bibliométrico y el análisis de redes de coautoría utilizando como herramienta base el software Pajek. De acuerdo con los resultados, México mantiene colaboración internacional desde los primeros años del estudio, aunque con menos intensidad durante los 80 y primera mitad de los 90 , donde predomina la

* CINVESTAV, México. meluna@cinvestav.mx

INVESTIGACIÓN BiBLIOTECOLÓGICA, Vol. 26, Núm. 57, mayo/agosto, 2012, México, ISSN: 0187-358X. pp. 103-129 
práctica de investigación en autoría única que está representada por el $40 \%$ del total de la producción. Es a partir de la segunda mitad de la década de los años noventa cuando México se integra con mayor fuerza a la colaboración internacional a través de 4,000 distintas instituciones procedentes de 143 diferentes países. El análisis de redes demuestra que es con Estados Unidos de América (EUA) con el que se logra una mayor colaboración científica, al igual que con Canadá e Inglaterra, lo anterior de acuerdo con las medidas de centralidad por grado, cercanía e intermediación. Finalmente, por el número de trabajos en cooperación, nuevamente destacan Estados Unidos, Canadá, Inglaterra, así como Italia, Alemania, Argentina, Cuba y Brasil.

Palabras clave: Ciencia en México; Colaboración científica; Internacionalización de la Ciencia.

\section{ABSTRACT \\ International scientific collaboration and recognition of Mexican science from 1980 to 2004 \\ María Elena Luna-Morales}

This paper presents an analysis of publications recorded in the Science Citation Index (SCI) databases from 1980 to 2004, and in the Social Science Citation Index (SSCI) databases from 1997 to 2004. In order to establish the level of international recognition of the Mexican science, we define in our study the basic aspects of the scientific collaboration with other institutions, countries and disciplines. This work is supported by the bibliometric method and co-authorship network analysis using Pajek software. In these results, Mexico has performed an international collaboration from the early years of the study, although with less intensity during the 80 's and the first half of the 90 's. I.e., during this period, the publishing schema has been dominated by the practice of research in single authorship, as evidenced by the fact that $40 \%$ of the total production was released without collaboration. In the second half of the 90's Mexico shows a stronger international collaboration through 4,000 different institutions from 143 different countries. The network analysis proves that Mexican collaboration with USA has the largest share, followed by those with Canada and England, in 
terms of the centrality measures of academic degree, proximity and mediators. Finally, according to the number of joint publications recorded in our study, the Mexican international collaboration is highlighted with United States, Canada, England, as well as with Italy, Germany, Argentina, Cuba and Brazil.

Keywords: Science in Mexico; Scientific Collaboration; Recognition of Mexican Science; International visibility of Mexican science

\section{INTRODUCCIÓN}

T a internacionalización científica es entendida como el resultado de un $ـ$ proceso que de manera espontánea e inducida tiene relevancia en la conformación y organización de las comunidades científicas. La internacionalización se puede medir a través de la producción y tecnología de conocimientos mediante sus mecanismos de difusión, transferencia y comercialización, así como en la interacción entre actores (Manual de Santiago, 2007).

Desde mediados del siglo XX, la colaboración es parte inherente de la investigación científica. Sin embargo, desde hace 20 años se están registrando cambios importantes con tendencia a crecimiento, relacionados con factores que conducen a los científicos a trabajar en equipo (Arvanitis, 2010). Por ejemplo, los que se relacionan con la especialización temática y la necesidad de afrontar problemas cada vez más complejos que hacen necesaria la intervención de grandes equipos de diferentes áreas de investigación para conformar espacios multidisciplinarios, interdisciplinarios y transdisciplinarios (Russell, Madera-Jaramillo y Ainsworth, 2009; Sancho, Morillo et al., 2006; Leydesdorff, 2006). En esta área la cooperación generalmente depende de lo abierta o cerrada que esté la comunidad científica para participar en proyectos de investigación con la misma o diferente institución del mismo o de distintos países; así como a interactuar con otras especialidades. El grado de colaboración depende del nivel del que se trate: local, regional, internacional, o bien, de carácter básico o aplicado.

La creciente importancia de la dimensión internacional es una de las características que definen la evolución de los modos de producción de conocimientos y de la organización de las actividades de investigación (Manual de Santiago, 2007). La apertura hacia la colaboración internacional está fuertemente relacionada con el idioma, la proximidad geográfica y la excelencia 
científica del país. No obstante, existen otras razones por las que se busca colaborar, tales como: 1) fuentes de financiamiento. Las organizaciones nacionales responsables de apoyar el desarrollo científico y tecnológico e incluso los organismos internacionales y fundaciones altruistas prefieren apoyar proyectos de investigación que se orientan a cubrir aspectos multidisciplinarios, donde se integran varios investigadores de distintas áreas para abordar problemas específicos; 2) complementar babilidades a través del cruce temático. Actualmente es muy común el desarrollo de investigaciones multidisciplinarias que buscan generar beneficios a la sociedad; 3) facilidad con las nuevas tecnologías de telecomunicaciones. Las telecomunicaciones ofrecen extraordinarias facilidades en el intercambio de información, sobre todo cuando han eliminado las fronteras geográficas para poner en contacto a los científicos de todo el mundo;(4) transferencia tecnológica industria-academia. Este aspecto es cada vez más promovido por los gobiernos, en particular por las ventajas que presenta al garantizar el desarrollo económico de cualquier nación; 5) las colaboraciones mejoran el progreso de la investigación. De acuerdo con Francis Macrina (2005), la colaboración permite a los investigadores hacer preguntas que no serían capaces de hacerse si trabajan en forma aislada, dando lugar a mejores resultados (Karlsson et al., 2008).

El tema sobre la colaboración científica no es algo nuevo, desde el momento en que se detectaron incrementos en el número de artículos co-escritos por investigadores de distintos países, también creció el interés por el desarrollo de estudios bibliométricos orientados a determinar los niveles en el incremento de las colaboraciones internacionales. En este sentido, algunos de los temas se enfocan a cubrir aspectos que tienen que ver con los factores que intervienen en la colaboración (Karlsson et al., 2008). En otros se trata de reflexiones sobre el mismo (Beaver, 2001; Miranda et al., 2010). Pero también se han dirigido esfuerzos al estudio de las redes de colaboración tomando como elemento base los grupos de investigación, los autores, las instituciones, las revistas y las áreas de investigación, así como el tipo de colaboración: local, regional e internacional (Sancho et al., 2006; Alonso-Arroyo, Pulgarín y Gil-Leiva, 2005; Russell, Ainsworth, y Narváez, 2006; Russell, 1998; Aguado-López et al., 2009); además de los estudios aplicados sobre la región asiática, donde los indicadores advierten un desempeño muy importante en la colaboración científica (Shin \& Cummings, 2010).

En este contexto de cambios en transición donde lo más sensato es involucrarse en lo que parece ser la lucha por el liderazgo de la ciencia y la tecnología (Beaver, 2001), México no está exento pues de igual manera contribuye en las pequeñas que en las grandes colaboraciones conocidas como Big Science, donde destacan los observatorios de astronomía, los aceleradores y 
detectores de partículas y los referentes al genoma humano (Luna-Morales y Russell, 2009; Noguera-Solano y Ruiz-Gutiérrez, 2000). Es por ello que el presente trabajo busca determinar la internacionalidad de la ciencia mexicana a partir de la colaboración científica establecida entre instituciones, países, continentes y disciplinas científicas a través de un análisis de la literatura científica registrada en las bases de datos Web of Science: Science Citation Index y Social Science Citation Index. De esta manera pretendemos probar que México siempre ha estado integrado a la colaboración científica internacional, actividad que se incrementó a principios de los años noventa, periodo en que se incorporaron a la ciencia nuevos esquemas de colaboración y publicación científica, dando como resultado trabajos más citados (Luna-Morales y Collazo-Reyes, 2005).

La bibliometría integra un conjunto de métodos que son aplicados para medir la colaboración científica, misma que pude ser cuantificable a partir de las publicaciones científicas firmadas por más de un autor, que pueden o no pertenecer a la misma institución (Russell, Madera-Jaramillo y Ainsworth, 2009; Glänzel y Schubert, 2001). En este sentido, son considerados indicadores de colaboración: los autores, las instituciones y los países que participan en el manuscrito, medidos con base en el número de documentos publicados conjuntamente por dos o más autores, instituciones y países (Alonso-Arroyo, Pulgarín y Gil-Leiva, 2005).

El estudio se apoya en el método bibliométrico que integra un conjunto de procedimientos para el análisis de la literatura científica publicada. Es importante aclarar que en la colaboración científica la medición se toma a partir de la coautoría registrada en los propios documentos publicados (autores, instituciones, países y disciplinas). El análisis de estas co-publicaciones trae como consecuencia la generación de indicadores de producción e impacto que ayuden a determinar los alcances de la cooperación internacional. Lo anterior en combinación con el análisis de redes de colaboración científica, tomando como herramienta base el software Pajek (Batajelj y Mrvar, 2010).

\section{MATERIALES Y MÉTODOS}

En las bases de datos Science Citation Index (SCI) y Social Science Citation Index (SSCI) se buscaron y recuperaron los trabajos publicados por instituciones mexicanas. La forma de búsqueda fue bajo dirección utilizando la estrategia MEXICO NOT (NEW MEXICO), cubriendo los periodos de búsqueda de 1980-2004 para SCI y de 1997-2004 en el caso de SSCI. Cabe aclarar que los cambios en los periodos de búsqueda se deben a los años adquiridos de las bases de datos propiedad de Thomson Reuters. 
En total de ambos índices se recuperaron 93,600 registros bibliográficos. Para asegurar que éstos pertenecían a instituciones de México se les aplicaron varios filtros que permitieron: 1) descartar trabajos duplicados, identificados a partir de la combinación de diversos campos, entre ellos: título, autor, fuente de publicación, volumen, páginas y año de publicación; 2) retirar registros que no pertenecían a instituciones mexicanas. Esto fue posible gracias a la desagregación que se aplicó a cada una de las direcciones incluidas en los trabajos, lo que permitió la integración de catálogos únicos e independientes por instituciones, dependencias, países, código postal, entre otros; 3) completar los campos de dirección de los registros donde no se incluía alguna o se mostraba incompleta. Para ello se utilizaron índices editados a nivel de diversos organismos e instituciones nacionales, así como las revistas impresas y en línea que conforman los fondos documentales de la Universidad Nacional Autónoma de México (UNAM) y el Centro de Investigación y Estudios Avanzados (Cinvestav). Por lo anterior, de un total de 93,600 registros extraídos de SCI y SSCI, únicamente se tomaron en cuenta para el análisis 79,443 , que son los que realmente pertenecen a instituciones mexicanas, mismos que fueron clasificados en siete apartados correspondientes a los continentes: 1) América-Norte, 2) Europa, 3) Asia, 4) África, 5) Oceanía, 6) América-Central y 7) América-Sur.

Para identificar los tipos de colaboración fue necesario llevar a cabo otra clasificación en la que se revisaron uno a uno los registros. Para facilitar el trabajo primero se apartaron los documentos que no presentaban esta característica, es decir, se trata de autorías únicas que en este estudio se van a identificar como sin colaboración (SC). Por otro lado, y según el número de países participantes en los trabajos, se fueron asignando las claves (CN) para los publicados únicamente por instituciones mexicanas (clasificación nacional); (CI) donde además de una o varias instituciones mexicanas también participan instituciones de otros países.

Por último, es importante aclarar que los nombres de los países se tomaron tal cual aparecen registrados en las bases de datos Web of Science. Es por ello que los casos de Inglaterra, Gales, Escocia e Irlanda del Norte que actualmente conforman el Reino Unido de la Gran Bretaña e Irlanda del Norte no se agruparon bajo este nombre, por el contrario aparecen de manera independiente. Lo mismo sucede con los nombres de otros países que también han sufrido cambios.

Por otro lado, las categorías temáticas Journal Citation Reports (JCR) asignadas a los trabajos publicados por instituciones mexicanas se catalogaron en 10 disciplinas científicas siguiendo el esquema que aplica el Atlas de la Ciencia Mexicana (http://www.atlasdelacienciamexicana.org/), entre ellas: 1) Agrociencias, 2) Ciencias Biológicas, 3) Ciencias Físicas, 4) Ciencias Químicas, 5) 
Ciencias Sociales, 6) Geociencias, 7) Humanidades, 8) Ingenierías, 9) Matemáticas y 10) Medicina y Ciencias de la Salud.

$\mathrm{El}$ análisis de redes de coautoría por países y continentes se construyó con base en dos matrices con sus respectivos vectores y particiones. Para ello fue indispensable llevar a cado la normalización de los datos, así como la separación y clasificación. Las matrices resultantes se ejecutaron utilizando la teoría de Pajek, y las redes se desarrollaron a niveles muy simples, es por eso que únicamente muestran la densidad de relación, la centralidad por grado, cercanía e intermediación, así como el tamaño en términos de producción científica.

Por último, para una mejor interpretación de los resultados, se aplicó el análisis de regresión a algunos de los datos, con la finalidad de proyectar los valores a partir de las líneas de tendencia particularmente exponencial y con los valores resultantes de las ecuaciones hacer interpretaciones con fundamento. Para calcular los porcentajes que en diversas tablas se muestran, se tomaron como parámetros base los totales de producción y citas registrados para México en las bases de datos Web of Science.

\section{Resultados}

\section{Aspectos generales del estudio}

Los índices del SCI y SSCI en el periodo 1980-2004 registran para México 79,443 trabajos y 532,289 citas únicas. Durante este tiempo se reconoce colaboración científica con 143 distintos países localizados a través de los diferentes continentes. Destaca la colaboración con naciones de Europa, Asia y África que se distinguen por el número de países que participan. No obstante que son pocos los territorios que conforman: América del Norte, América Central, América del Sur y Oceanía, son muy importantes las contribuciones que tienen con México, sobre todo Estados Unidos y Canadá, así como Brasil, Argentina, Chile, Cuba y Colombia.

Para dar mayor claridad en los resultados que se muestran en este apartado, se expone por medio de la Tabla 1 la distribución de trabajos y citas para la ciencia mexicana en el periodo de 1980-2004. Como se muestra, durante la primera década México se sostuvo con una producción que no rebasó los 2000 trabajos. A comienzos del periodo de los años noventa se registran crecimientos importantes que se duplican en comparación con el periodo anterior. No obstante a partir del año 2000 se infieren incrementos igualmente considerables y con tendencias nuevamente a duplicarse y a sostener estos crecimientos en el tiempo. El comportamiento de las citas es muy semejante al de 
los trabajos, a pesar de que en los últimos años se muestran decrementos muy fuertes, sin embargo, esta es una situación normal ya que los documentos publicados en los últimos años no han recuperado el total de citas.

Tabla 1. Trabajos y citas por serie anual

\begin{tabular}{|c|c|c|}
\hline Años & Trabajos & Citas \\
\hline 1980 & 1209 & 11659 \\
\hline 1981 & 1355 & 13524 \\
\hline 1982 & 1019 & 10549 \\
\hline 1983 & 1164 & 11185 \\
\hline 1984 & 1217 & 11667 \\
\hline 1985 & 1251 & 13981 \\
\hline 1986 & 1397 & 12566 \\
\hline 1987 & 1530 & 14089 \\
\hline 1988 & 1691 & 14524 \\
\hline 1989 & 1521 & 18285 \\
\hline 1990 & 1649 & 18983 \\
\hline 1991 & 1891 & 16387 \\
\hline 1992 & 2046 & 23641 \\
\hline 1993 & 2420 & 27185 \\
\hline 1994 & 2629 & 25626 \\
\hline 1995 & 3002 & 31071 \\
\hline 1996 & 3871 & 35451 \\
\hline 1997 & 4377 & 36052 \\
\hline 1998 & 4657 & 36142 \\
\hline 1999 & 5558 & 39515 \\
\hline 2000 & 5649 & 35669 \\
\hline 2001 & 6284 & 31994 \\
\hline 2002 & 6385 & 12631 \\
\hline 2003 & 7156 & 18637 \\
\hline \multirow[t]{2}{*}{2004} & 8505 & 11276 \\
\hline & 79433 & 532289 \\
\hline
\end{tabular}

Del total de trabajos publicados en el periodo analizado, $40 \%$ corresponde a trabajos sin colaboración (SC), 22\% a colaboración nacional (CN) y 38\% a internacional (CI).

La Figura 1 presenta por quinquenios la evolución de los tipos de colaboración. Como se puede observar, en los primeros quinquenios de los años ochenta predomina la práctica de publicación en autoría única, es decir, sin colaboración (SC). En el segundo quinquenio se iguala la participación SC y CI, y es a partir del tercer quinquenio cuando se modifica la situación porque se empieza a 
imponer la colaboración de tipo internacional, a la vez que comienzan a descender las contribuciones SC. La colaboración nacional es la única que se sostiene durante los tres primeros periodos por debajo de las demás, y es hasta el cuarto quinquenio cuando se empareja con las publicaciones SC y termina rebasando tal como se muestra en el último quinquenio. Esta misma situación la manifiesta la CI que desde el quinquenio 1900-1994 crece de manera exponencial.

Los valores de las ecuaciones resultantes de la incorporación de líneas de tendencia exponencial indican que la constante de crecimiento para los trabajos SC es menor que las demás al alcanzar un crecimiento de 0.27 en el periodo analizado, con un ajuste de crecimiento de R2=0.931 y que de acuerdo con la forma en que estos datos se interpretan, entre más se acercan a 1 es muestra de crecimientos muy aceptables.

$\mathrm{La} \mathrm{CN}$ que se inicia con un promedio de 423 trabajos en los primeros años del estudio se sostiene con crecimientos constantes de 0.77 , en tanto que el valor de regresión es de $\mathrm{R} 2=0.985$, lo que demuestra un ajuste de crecimiento muy bueno. Algo parecido presenta la CI, la variante estriba en que ésta registra el mejor ajuste de crecimiento $\mathrm{R} 2=0.972$ de las tres variables analizadas. De hecho es la CI la que se duplica en un lapso de tres periodos: 1990-1994, 19951999 y 2000-2004. En conclusión los tres tipos de colaboración registran crecimientos constantes, incluso los trabajos SC que reportan una leve caída en el último periodo, lo que puede significar dos situaciones: (1) que la comunidad científica en México se está inclinando cada vez más por el trabajo en coautoría, (2) es una consecuencia de las políticas científicas aplicadas en este país.

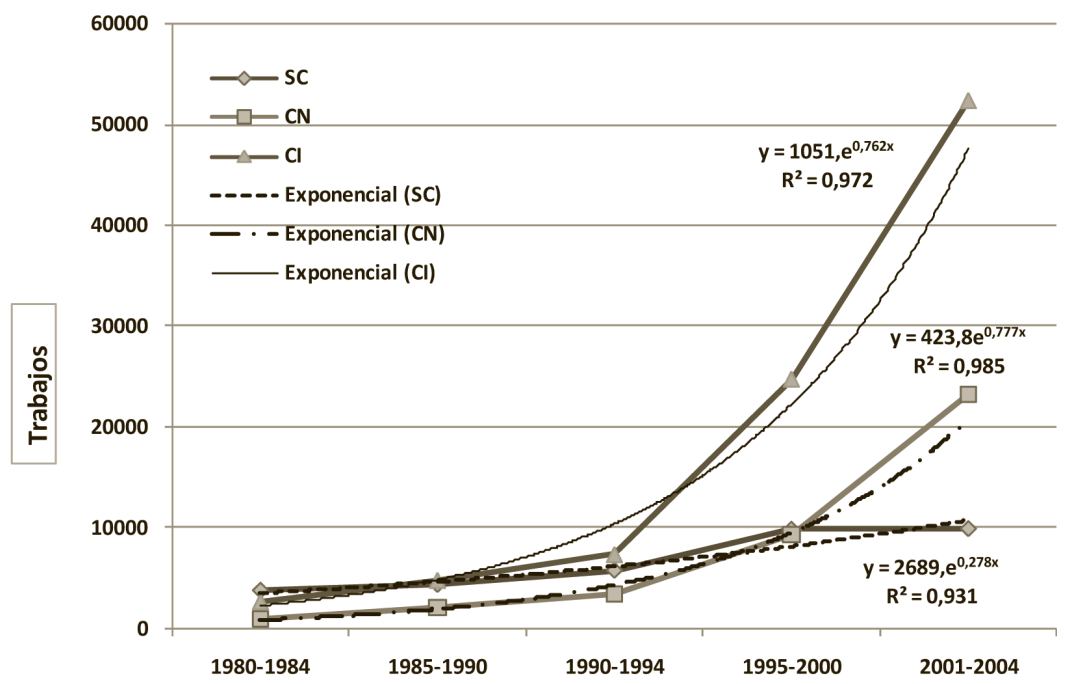

Fig. 1. Evolución por quinquenio de los tipos de colaboración científica 


\section{México en colaboración con otros países}

Los 21 países que más colaboración científica registran con México son mostrados en la Tabla 2, misma que integra dos numeraciones independientes y cada una está ordenada en forma ascendente para indicar el ranking de ubicación de los países; el segundo orden es descendente por trabajos y citas. Los porcentajes de trabajos y citas se obtienen del global de trabajos y citas registrados en las bases de datos Web of Science con adscripción a México.

De acuerdo con los resultados, México sostiene una importante colaboración con Estados Unidos con el que consigue publicar 8.4\% del total de los trabajos, los que a su vez logran 3.9\% del global de las citas acumuladas para nuestro país. España es otra de las naciones con la que también se consigue una importante participación científica, con este país sostiene $3.7 \%$ en publicaciones y 3.4\% de citas. Las variantes en los porcentajes es la razón por la que no conserva el mismo ranking en ambos arreglos. Los únicos países que sí conservan ambas posiciones son Estados Unidos, Francia y Alemania; esto indica que la visibilidad de los trabajos es lo que hace la diferencia, porque puede publicar menos trabajos por ejemplo con Brasil, sin embargo, las citas que se recuperan de estos documentos son mayores que los que se dieron a conocer con España, Francia, Inglaterra entre otros países que registran mayor número de trabajos en colaboración.

Es importante destacar que a nivel de países de América Central y América del Sur sobresale la colaboración con Cuba, Brasil, Argentina, Colombia y Chile que en conjunto consiguen el $4.4 \%$ de los trabajos en colaboración y $5.6 \%$ de las citas. En general, estos 21 países registran 35\% del total de las publicaciones, y $38 \%$ de citas. Lo que quiere decir que el grueso de la colaboración la mantiene con los 122 países restantes. Esto implica que en promedio son aproximadamente 414 trabajos los que se tienen en colaboración con cada una de estas naciones, un equivalente en citas a 2,455.

Tabla 2. Países que registran la más alta colaboración científica con México

\begin{tabular}{|c|l|c|c|c|l|c|c|}
\hline Núm. & \multicolumn{1}{|c|}{ País } & Trabajos & \% Trabajos & Núm. & \multicolumn{1}{|c|}{ País } & Citas & $\%$ Citas \\
\hline 1 & $\begin{array}{l}\text { Estados Unidos } \\
\text { (EUA) }\end{array}$ & 6656 & 8.4 & 1 & $\begin{array}{l}\text { Estados Unidos } \\
(\text { EUA })\end{array}$ & 20911 & 3.9 \\
\hline 2 & España & 2923 & 3.7 & 2 & Inglaterra & 18336 & 3.4 \\
\hline 3 & Francia & 2795 & 3.5 & 3 & Francia & 17904 & 3.4 \\
\hline 4 & Inglaterra & 2168 & 2.7 & 4 & Canadá & 15311 & 2.9 \\
\hline 5 & Alemania & 1851 & 2.3 & 5 & Alemania & 14102 & 2.6 \\
\hline 6 & Canadá & 1683 & 2.1 & 6 & Brasil & 11151 & 2.1 \\
\hline 7 & Rusia & 1135 & 1.4 & 7 & Italia & 10464 & 2.0 \\
\hline
\end{tabular}




\begin{tabular}{|c|l|c|c|c|l|c|c|}
\hline 8 & Italia & 1061 & 1.3 & 8 & Suiza & 9433 & 1.8 \\
\hline 9 & Brasil & 1029 & 1.3 & 9 & Holanda & 8373 & 1.6 \\
\hline 10 & Argentina & 756 & 1.0 & 10 & España & 8179 & 1.5 \\
\hline 11 & Cuba & 731 & 0.9 & 11 & Bélgica & 6788 & 1.3 \\
\hline 12 & Japón & 659 & 0.8 & 12 & Chile & 6667 & 1.3 \\
\hline 13 & Polonia & 527 & 0.7 & 13 & Noruega & 6375 & 1.2 \\
\hline 14 & Colombia & 500 & 0.6 & 14 & Rusia & 6363 & 1.2 \\
\hline 15 & Chile & 495 & 0.6 & 15 & Dinamarca & 6325 & 1.2 \\
\hline 16 & Holanda & 477 & 0.6 & 16 & Suecia & 6121 & 1.1 \\
\hline 17 & Suiza & 475 & 0.6 & 17 & Colombia & 6027 & 1.1 \\
\hline 18 & India & 470 & 0.6 & 18 & Sudáfrica & 5696 & 1.1 \\
\hline 19 & Bélgica & 468 & 0.6 & 19 & Argentina & 5606 & 1.1 \\
\hline 20 & Australia & 468 & 0.6 & 20 & Japón & 5293 & 1.0 \\
\hline 21 & RP China & 460 & 0.6 & 21 & Israel & 5187 & 1.0 \\
\hline & 122 restantes & 50556 & 65.0 & & 122 restantes & 299598 & 62.0 \\
\hline
\end{tabular}

\section{Nivel de colaboración}

En la Figura 2 se muestra en porcentajes el nivel de cooperación según el número de países que intervienen en la elaboración de los manuscritos. Los datos son muy claros, en estos se advierten tendencias en la cooperación bilateral y trilateral donde sólo participan dos y tres países distintos. La participación entre cuatro países es otro de los datos sobresalientes, al igual que las cooperaciones donde participan más de 10 naciones. En estas últimas están ubicadas las colaboraciones Big Science en las que México tiene participación a través de áreas como la física de campos y partículas, biomedicina molecular y astro-partículas. Finalmente, con menos de 10\% de cooperación están las distribuidas entre cinco y diez países. 


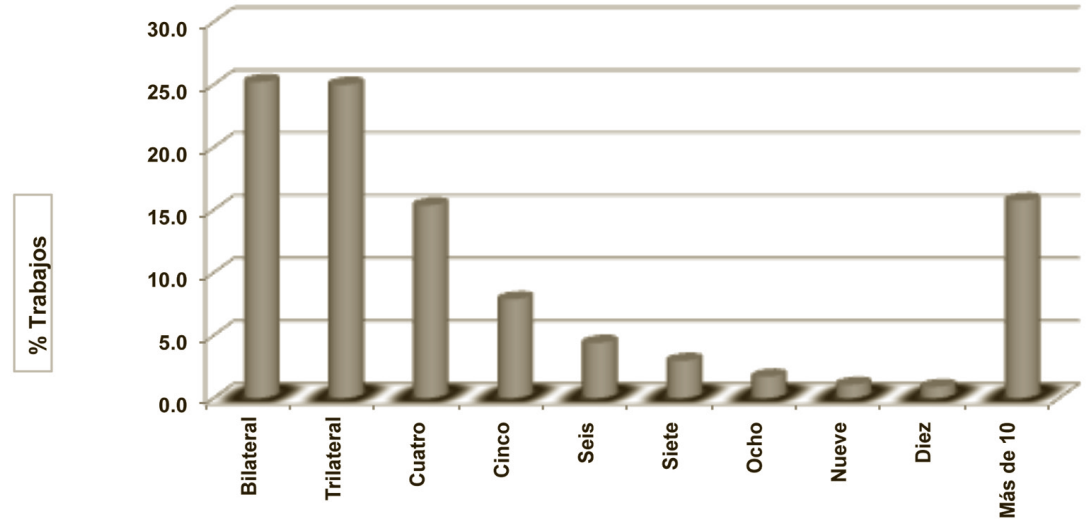

Nivel de colaboración

Fig. 2. Distribución de trabajos por nivel de colaboración

Según el análisis de redes de colaboración por países observamos que el número de vértices analizados son 143 , y el total de líneas que se generan 1 078.

La Tabla 3 hace referencia a los 10 países que registran el mayor grado de centralidad según los siguientes tipos: por grado, por cercanía y por intermediación. En este sentido, la centralidad por grado favorece a Estados Unidos, Alemania, Italia y Francia que tienen altos niveles de colaboración. Los países que manifiestan menor grado de centralidad son: Argentina y Australia.

La centralidad por cercanía indica que Canadá es el país que tiene el grado más alto; el segundo lugar le corresponde a Austria e Inglaterra, el tercero es para Argentina y Bélgica. Brasil está en la cuarta posición y el resto de los países, por sus valores, están ubicados en los últimos lugares entre los 10 principales. Finalmente los países que están mejor posicionados con respecto a la centralidad por intermediación, es decir, que son vínculo para conectar a otros países son: Inglaterra y Canadá, al igual que Italia y Alemania. 


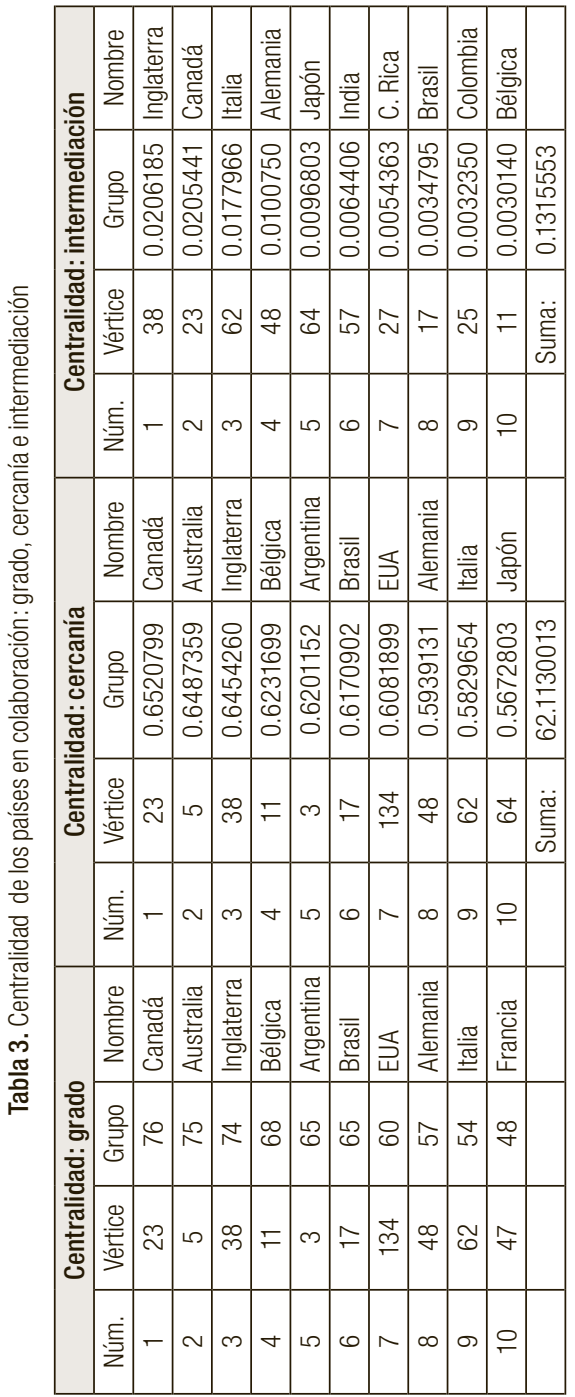


Los países clasificados por trabajos en colaboración son mostrados en la Figura 3. Como se observa, están organizados en varios niveles según el número de trabajos que sostienen en cooperación. En este caso, el algoritmo de partición que aplica Pajek reporta la presencia de cinco grupos: el primero conformado por los países que reúnen de 1 a 50 trabajos en colaboración, constituido por 116 países, lo que equivale al $80 \%$ del total de los mismos, mostrados en la gráfica por las esferas clasificadas con el número 1. El segundo grupo está compuesto por siete países que en colaboración reportan de 50 a 100 trabajos, se reconocen por el número 2 de partición. El tercero está integrado por 13 países, señalados con el número 3, y mantienen en colaboración de 100 a 500 trabajos. El cuarto y quinto grupos están conformados ambos por cuatro países, el primero de ellos comparte en colaboración 500 y 1000 trabajos y están representados por las esferas con el número 4; el segundo clasificado con el número 5 publica entre 1,000 y 1,625 documentos. Como se muestra, los países que mantienen más trabajos en colaboración están concentrados en el centro de la figura, mientras que los de la periferia son los que menos cooperación sostienen. 


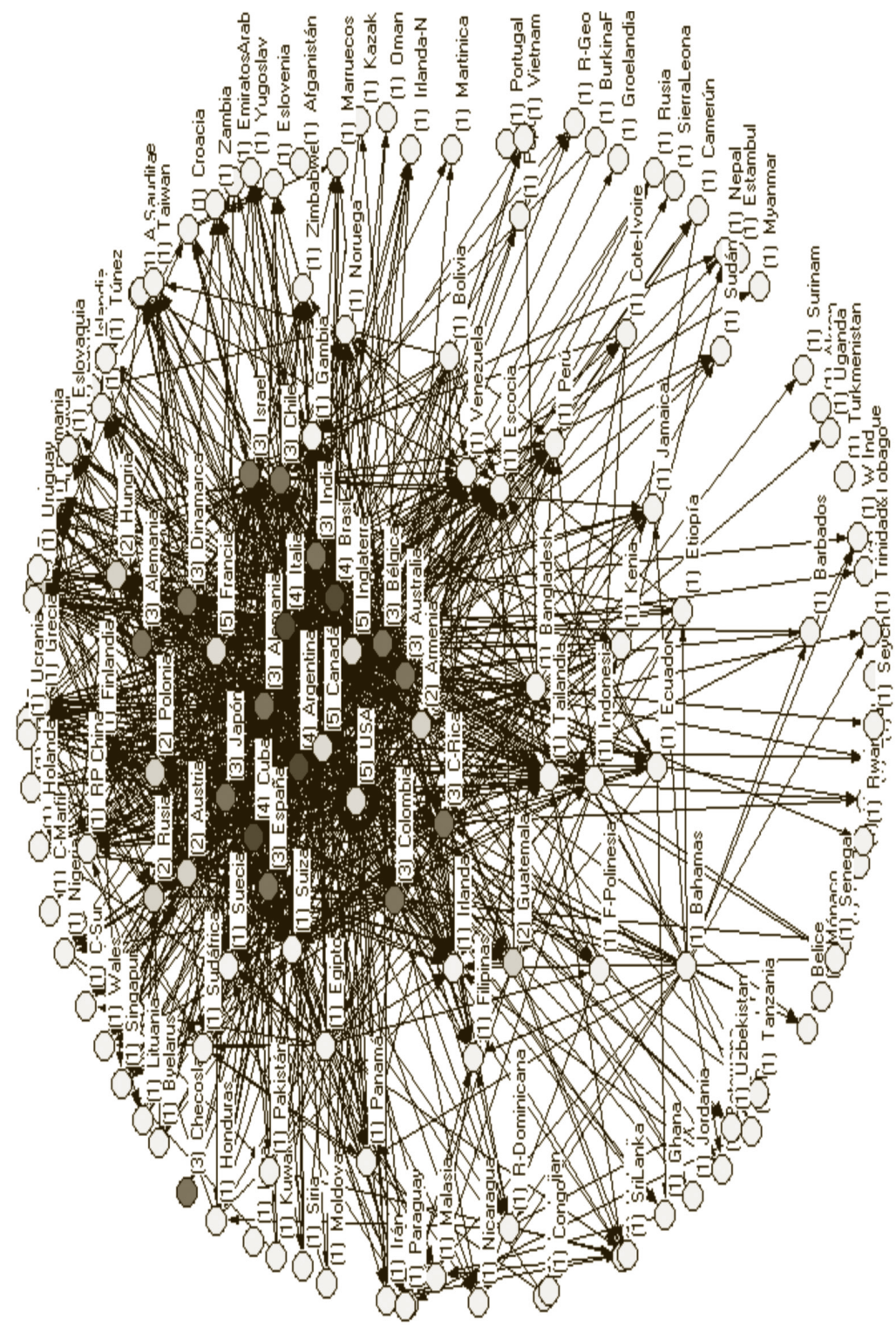

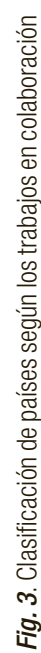


La Tabla 4 hace referencia a las 26 instituciones que más alta colaboración científica registran con dependencias mexicanas. Como se muestra es con la University of Texas con la que más participación se tiene, 1.1\% del total de la producción registrada para México. A través del Consejo Superior de Investigaciones Científicas (CSIC) se publica $0.9 \%$. En este orden de coautoría institucional por trabajos le siguen: la Universidad de la Habana con $0.9 \%$, University of Arizona $0.7 \%$, así como University of California Davis $0.6 \%$; con University of Texas A\&M se reconoce $0.5 \%$. Como lo muestra la lista, también sobresalen University of California Berkeley, University Harvard, CNRS, Universidad Autónoma de Barcelona, entre otras, con las que sostiene colaboraciones que varían entre 308 y 378 trabajos. El resto de las instituciones $(4,254)$ muestra colaboración con instituciones mexicanas publicando menos de 300 trabajos, no obstante, juntas registran el $87.5 \%$ de los trabajos publicados.

La misma Tabla presenta a las instituciones que destacan por el alto número de citas, entre ellas, University of Texas y University of Harvard que $\log$ ran $1.4 \%$ del global. University of Michigan es la tercera más representativa al conseguir el 1.1\%. Las demás instituciones presentan cooperación con menos del 1\% de citas. En términos generales estas 26 dependencias recogen únicamente el 13\% del total de los trabajos, el 21\% de las citas. Lo que quiere decir que la colaboración está distribuida entre un gran número de instituciones $(4,254)$ que en conjunto logran $87 \%$ y $79 \%$ de los totales.

Por último, y como se puede ver, gran parte de las instituciones se localizan en Estados Unidos, muy pocas corresponden a Inglaterra, Canadá, Francia, España, entre otros países. En América Latina y el Caribe las únicas colaboraciones fuertes ocurren con la Universidad de la Habana, Cuba y el Instituto de Investigaciones en Medicina Naval de Perú. 


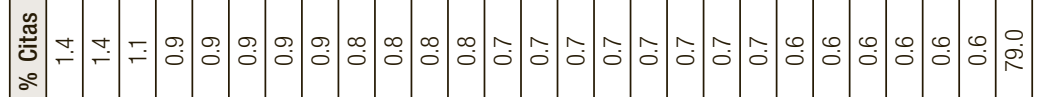

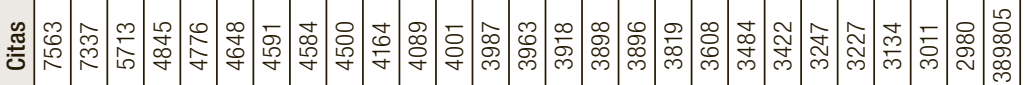

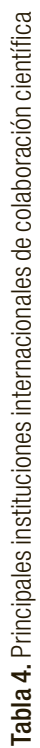

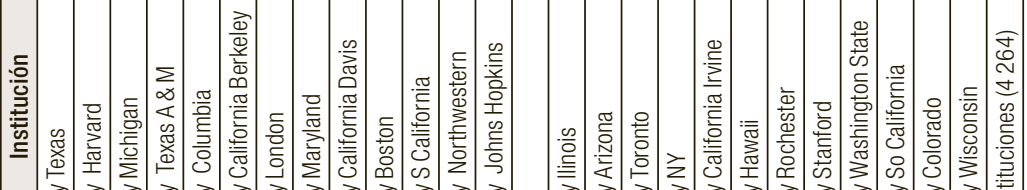

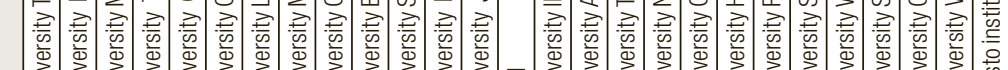
竞竞

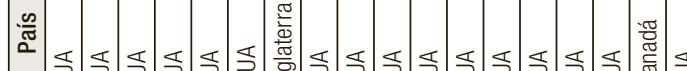

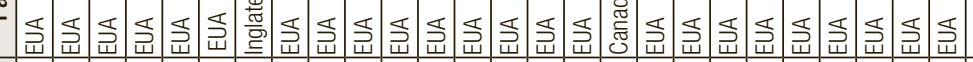

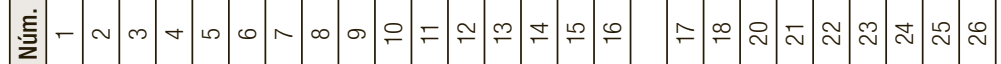

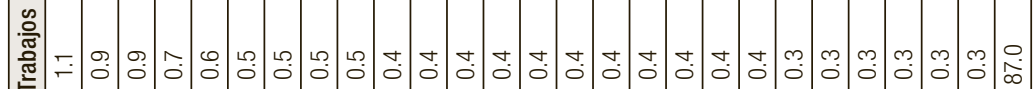

它

$\therefore$

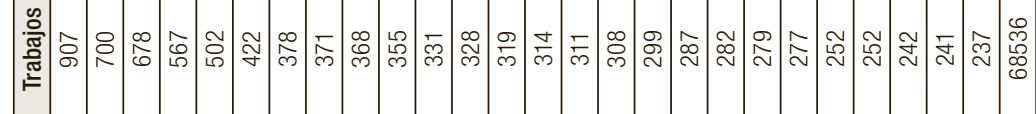

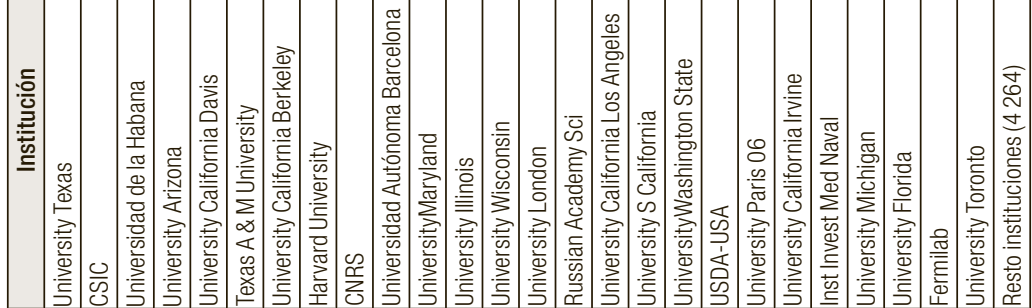

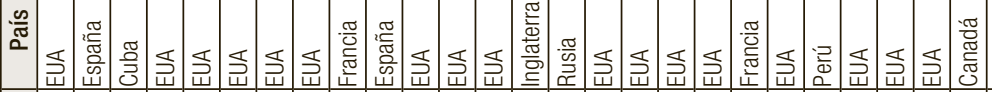

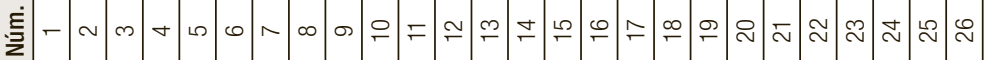


La Tabla 5 presenta la colaboración por disciplina científica, los datos estás estructurados en cuatro apartados: 1) descripción de la disciplina, 2) número de países en colaboración por disciplina, 3) trabajos que se comparten en la colaboración, 4) porcentajes de acuerdo con el total de trabajos registrados para México en Web of Science.

Como se advierte, medicina y ciencias de la salud es la disciplina que más naciones atraen en cooperación: 99 de las 143 identificadas en el periodo estudiado, con estos países se publican 18,055 trabajos de investigación, equivalentes a $238 \%$ del total de trabajos registrados para México. De igual manera destacan ciencias biológicas e ingenierías, la primera reúne a 94 países con los que da a conocer 13,763 trabajos y estos representan $17 \%$ de los trabajos publicados; la segunda agrupa a 79 países y en conjunto difunden 8,603 (11\%) trabajos. El resto de las disciplinas, como se advierte, también tienen una participación destacada, tal es el caso de las ciencias físicas que registran colaboración con 80 países y es la disciplina que mayor número de trabajos publica por esta vía $(22,530)$, equivalente al $28 \%$ de los documentos. Es muy probable que estas disciplinas destaquen más que las otras porque se trata de áreas de investigación con amplia trayectoria científica en el país. Pero también nos referimos a áreas que se incorporaron desde principios de los años noventa a las grandes ciencias, como la física de partículas y campos, genómicas y astropartículas.

Las humanidades tienen muy baja colaboración, al igual que las matemáticas y las ciencias sociales. Sin embargo, ésta es una característica de estas disciplinas que orientan sus esfuerzos hacia temas de investigación endógena. Es importante aclarar que las humanidades no están adecuadamente representadas dado que los indicadores mostrados en este trabajo corresponden a documentos que aparecen registrados únicamente en los índices de ciencias y ciencias sociales.

En conclusión, la colaboración científica está más representada a través de disciplinas como: medicina y ciencias de la salud, ciencias físicas, ciencias biológicas e ingenierías que son las que reportan mayor número de trabajos. No obstante, por el número de países con los que colabora, también se suman: agrocienicas, ciencias químicas y de la tierra.

Tabla 5. Colaboración científica internacional por disciplina científica

\begin{tabular}{|l|l|l|}
\hline Disciplina científica & Países en & Trabajos en
\end{tabular}

\begin{tabular}{|c|l|c|c|c|}
\hline Núm. & \multicolumn{1}{|c|}{ Disciplina científica } & $\begin{array}{c}\text { Países en } \\
\text { colaboración }\end{array}$ & $\begin{array}{c}\text { Trabajos en } \\
\text { colaboración }\end{array}$ & $\begin{array}{c}\text { \% Trabajos } \\
\text { colaboración }\end{array}$ \\
\hline 1 & Medicina y Ciencias de la Salud & 99 & 18055 & 23 \\
\hline 2 & Ciencias Biológicas & 94 & 13763 & 17 \\
\hline 3 & Ingenierías & 79 & 8603 & 11 \\
\hline
\end{tabular}




\begin{tabular}{|c|l|c|c|c|}
\hline 4 & Ciencias Físicas & 80 & 22530 & 28 \\
\hline 5 & Agrociencias & 63 & 1822 & 23 \\
\hline 6 & Ciencias Químicas & 66 & 4330 & 5 \\
\hline 7 & Ciencias de la Tierra & 45 & 3120 & 39 \\
\hline 8 & $\begin{array}{l}\text { Ciencias Sociales y Ciencias del } \\
\text { Comportamiento }\end{array}$ & 17 & 2020 & 3 \\
\hline 9 & Matemáticas & 45 & 2800 & 35 \\
\hline 10 & Humanidades & 10 & 2400 & 3 \\
\hline & & 143 & 79443 & 100 \\
\hline
\end{tabular}

\section{Colaboración por categoría temática}

La Tabla 6 presenta las categorías temáticas que registran mayor participación en la colaboración científica entre países. Como se observa únicamente se muestran las categorías que presentan porcentajes iguales o superiores al $0.4 \%$ de trabajos en cooperación. Las áreas físicas son las que sobresalen por la colaboración que sostienen principalmente con Estados Unidos, Francia, Rusia, Alemania, Italia y Brasil. La astronomía y astrofísica es otra de las áreas que presentan colaboración sobre todo con países como: Estados Unidos, España, Francia, Inglaterra, Italia y Alemania. Nuevamente con Estados Unidos sobresale la participación científica a través de las categorías de Medicina, General e Interna; Salud Pública Ambiental y Ocupacional, Ciencia de las Plantas, Agronomía, Genética y Herencia. El hecho de que destaquen las áreas físicas al igual que astronomía y astrofísica tiene mucho que ver con la apertura de varios proyectos de colaboración establecidos a nivel internacional, donde destacan los orientados a la física de partículas y campos y astronomía y astrofísica. En éstos participan instituciones como la Universidad Nacional Autónoma de México (UNAM), el Centro de Investigación y de Estudios Avanzados (Cinvestav), además del Instituto Nacional de Astrofísica, Óptica y Electrónica (INAOE) como también se muestra en la Tabla 7. En términos generales estas categorías representan el 16\% del total de las citas. Lo que quiere decir, que el $84 \%$ restante se divide entre una amplia variedad de temas de investigación.

Tabla 6. Porcentajes de colaboración por países y categorías temáticas

\begin{tabular}{|l|l|c|c|}
\hline \multicolumn{1}{|c|}{ País } & \multicolumn{1}{c|}{ Categorías temáticas } & Trabajos & \% trabajos \\
\hline EUA & Physics, Multidisciplinary & 3872 & 4.9 \\
\hline EUA & Astronomy \& Astrophysics & 1271 & 1.6 \\
\hline EUA & Physics, Particles \& Fields & 1228 & 1.5 \\
\hline España & Astronomy \& Astrophysics & 550 & 0.7 \\
\hline EUA & Multidisciplinary Sciences & 480 & 0.6 \\
\hline Francia & Physics, Multidisciplinary & 441 & 0.6 \\
\hline
\end{tabular}




\begin{tabular}{|l|l|c|c|}
\hline Rusia & Physics, Multidisciplinary & 417 & 0.5 \\
\hline Francia & Astronomy \& Astrophysics & 411 & 0.5 \\
\hline Inglaterra & Astronomy \& Astrophysics & 373 & 0.5 \\
\hline EUA & Medicine, General \& Internal & 373 & 0.5 \\
\hline EUA & Plant Sciences & 360 & 0.5 \\
\hline Alemania & Physics, Multidisciplinary & 354 & 0.4 \\
\hline Alemania & Physics, Particles \& Fields & 349 & 0.4 \\
\hline EUA & Genetics \& Heredity & 343 & 0.4 \\
\hline Alemania & Astronomy \& Astrophysics & 329 & 0.4 \\
\hline EUA & Public, Environmental \& Occupational Health & 324 & 0.4 \\
\hline Italia & Astronomy \& Astrophysics & 323 & 0.4 \\
\hline Brasil & Physics, Multidisciplinary & 309 & 0.4 \\
\hline Francia & Physics, Particles \& Fields & 307 & 0.4 \\
\hline & & 12414 & 16 \\
\hline
\end{tabular}

En la Tabla 7 se señalan las instituciones mexicanas que presentan mayor colaboración científica según las categorías temáticas en que son clasificadas las revistas de publicación de los trabajos. Como se advierte, hay mucha relación con la tabla anterior, más aún cuando destaca la UNAM como una de las instituciones con más colaboración por medio del área de astronomía y astrofísica, al igual que la física multidisciplinaria donde quedan clasificados gran parte de los trabajos de física de partículas y campos, donde el Cinvestav también es un fuerte colaborador. No obstante, también destacan la Secretaría de Salud (SSA) y el Instituto Mexicano del Seguro Social (IMSS) por las áreas de Medicina general e interna, Medicina, investigación y Experimentación, Reumatología y Gastroenterología y Hepatología. A través de la UNAM sobresalen otros campos de investigación como: química, neurociencias, ciencias de las plantas, matemáticas, entre otras. El INAOE y el Centro de Investigación en Óptica son otras dependencias que destacan por la cooperación que registran en el área de Astronomía y astrofísica, así como óptica.

Con respecto a las citas por categoría temática advertimos que sigue siendo Astronomía y Astrofísica la que mejor posicionada queda, gracias al trabajo que se realiza en este campo en la UNAM y en el INAOE; juntas, estas instituciones consiguen $3.0 \%$ del total de las citas. La SSA por otro lado a través de Medicina General e Interna logra 1.6\% de las mismas. Una situación similar presenta Neurociencias y las áreas físicas, ambas desarrolladas en la UNAM y en el Cinvestav. En conjunto, estas categorías e instituciones alcanzan aproximadamente $2 \%$ y $4 \%$, respectivamente, del total de las citas. El resto de las categorías mostradas en la Tabla 7 obtienen porcentajes que varían entre $0.4 \%$ y $1.0 \%$ donde son representativas las áreas de Reumatología, Geoquímica y Geofísica, Microbiología y Agrociencias, entre otras. 


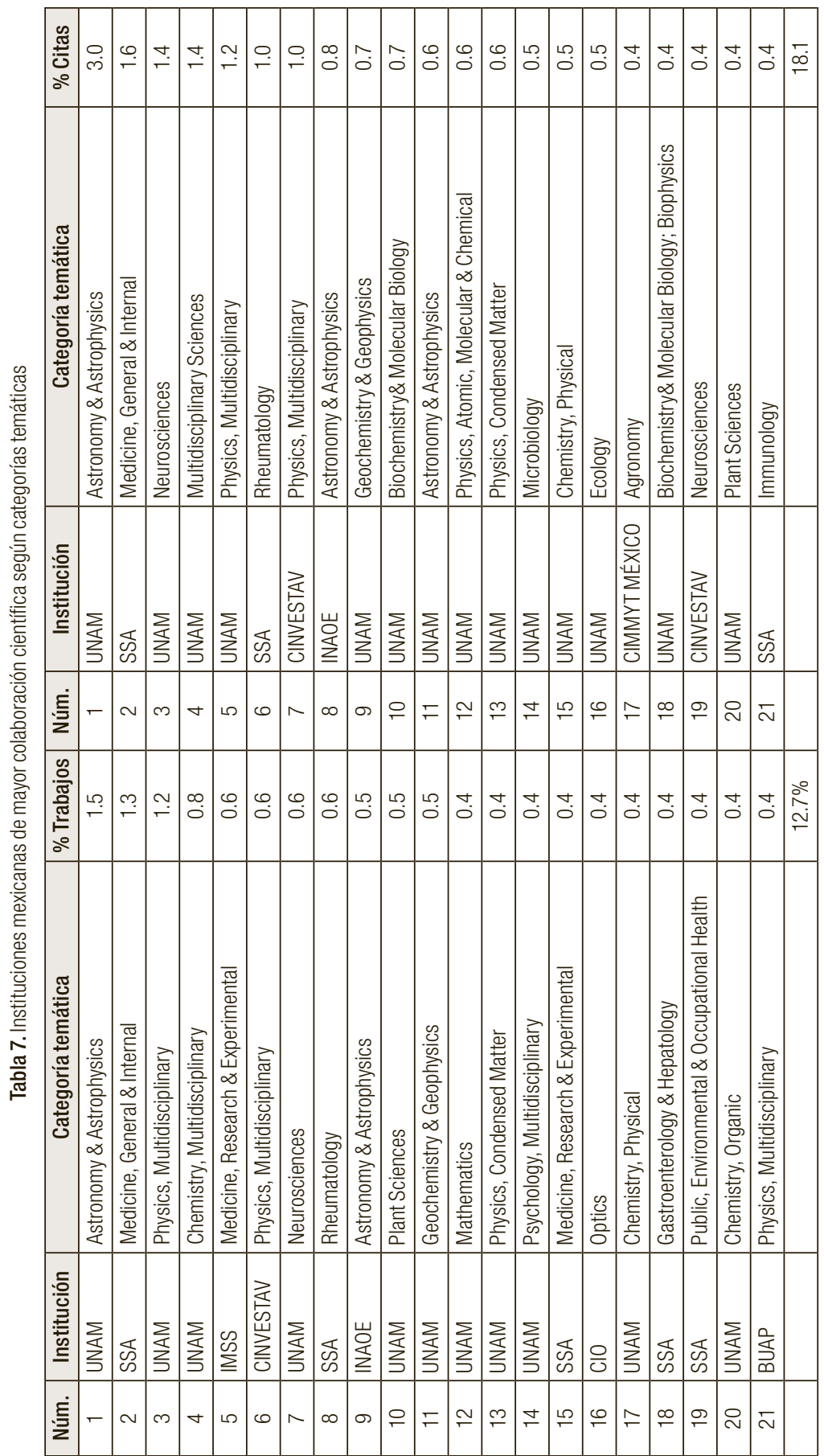


La investigación científica en México está evolucionando, adaptándose a las condiciones de la publicación universal establecida por la ciencia moderna, siguiendo un esquema que cada vez se aleja más del significado del contexto local en la producción de conocimiento (Somsen, 2008). Sin embargo, este acoplamiento ha sido lento como lo demuestra el hecho de que poco más del $40 \%$ de la producción de corriente principal registrada para México se ha realizado en forma individual, sobre todo la difundida durante la década de los años ochenta y principios de los noventa. No obstante, en el periodo de los noventa se comienzan a notar los primeros incrementos significativos y constantes en la colaboración científica internacional. De alguna manera esto tiene que ver con la generación de nuevas formas de hacer ciencia como un modo de influir en los procesos de maduración de las áreas de investigación y como una manera de aprovechar los recursos económicos, humanos, materiales y de información (Luna-Morales y Collazo-Reyes, 2002; Luna-Morales y Russell, 2009; Noguera-Solano y Ruiz-Gutiérrez, 2000).

La cooperación más fuerte entre instituciones y países está ligada principalmente hacia Estados Unidos y España, lo cual no es ninguna novedad, sobre todo cuando Estados Unidos geográficamente colinda al norte con México, lo que sin duda es una gran ventaja para la comunidad científica de este país. Por otro lado, Estados Unidos es el país número uno en el mundo, el que tiene las mejores condiciones para realizar investigación científica y tecnológica. Es por ello que en el análisis de redes despunta la colaboración con esta nación particularmente en la centralidad por grado, es decir, es el país con el que mayor colaboración se registra no sólo a nivel de países, sino también de instituciones y categorías temáticas.

España es el otro país que sostiene amplia cooperación científica con México. Sin duda, uno de los aspectos que identifica a estos países es el idioma, así como el interés por el desarrollo de proyectos en conjunto, en particular aquellos que funcionan con base en recursos compartidos, característica que el Consejo Nacional de Ciencia y Tecnología (CONACyT) toma mucho en cuenta en el establecimiento de proyectos de colaboración, facilidades que España garantiza y que quizás sea la razón por la que se logran convenios importantes para diferentes disciplinas como física, medicina y ciencias de la salud, entre otras.

Otro de los aspectos que seguramente tiene que ver con los cambios ocurridos en la colaboración científica es la especialización del conocimiento. Históricamente, estos acontecimientos se originaron en años posteriores a la Segunda Guerra Mundial y se intensificaron durante los años noventa (Leydesdorff, 2006), periodo en el que se sobreponen varios motivos, entre 
otros, el desarrollo de grandes proyectos de investigación y el incremento en los costos de la misma, la construcción de las herramientas de laboratorios cada vez más ligadas al desarrollo científico tecnológico y el acceso a los recursos de información. En este caso, forman parte de las tendencias de colaboración los convenios establecidos por medio de disciplinas y áreas de investigación donde México sostiene un alto número de convenios de nivel internacional. De acuerdo con las bases de datos Web of Science, México registra más de 100 proyectos de cooperación en grupo, es decir, se trata de grandes colaboraciones. En este sentido, y por las ventajas que ofrece la publicación basada en la colaboración científica, es conveniente que México sostenga, y en lo posible incremente, el número de proyectos y convenios de participación con otras instituciones y países. Más aún cuando la OCDE estableció que la colaboración nacional e internacional es de extrema importancia para facilitar el desarrollo sostenible, así como favorecer la movilidad de los investigadores y aumentar el acervo cultural y científico de los participantes (Sancho et al., 2006; Organization of American States, 2006). Además de que dan lugar a trabajos altamente citados entre los que destacan los pertenecientes a las disciplinas: físicas, biológicas, ingenierías, medicina y ciencias de la salud. Es precisamente a través de estas áreas, incluyendo las agrociencias e ingenierías, que México ha logrado un gran avance en la internacionalización de la ciencia por medio de la colaboración que sostiene con diferentes instituciones de distintos países. Esta misma situación es mostrada por J.M. Russell et al. (2008) y Pillaid K.G. (2007), que señalan como ha ascendido la colaboración en las áreas de ciencias físicas, ingenierías y matemáticas.

En conclusión, en los últimos diez años México ha incrementado la colaboración científica internacional como consecuencia de varias situaciones ocurridas y que son consideradas determinantes en este proceso:

- Aplicación de políticas científicas mediante las cuales el Conacyt tiene prioridad para apoyar proyectos de investigación de alcance científico mundial, ya que ésta es una forma de compartir gastos con otros países. En este esquema de cooperación aparece registrada la participación de áreas como: física de partículas y campos, astronomía y astrofísica, genética, biología molecular y algunos campos de la medicina (Aretxaga, 2008; Rivera, 1999).

- Iniciativas de la propia comunidad científica, que por este medio buscan implementar proyectos de investigación en conjunto con investigadores de otros países e instituciones. En algunas ocasiones porque persiguen objetivos comunes que tienen que ver con aplicaciones en áreas muy específicas. 
- Proyectos de cooperación internacional establecidos a partir de acuerdos con organizaciones internacionales como la Organización Mundial de la Salud (OMS) y Unesco que buscan desarrollar investigación en campos específicos que afectan a países en vías de desarrollo. En estos casos se trata generalmente de progresos enfocados a cubrir temas de investigación local y regional, donde la cooperación es a nivel de países de América Central y del Caribe y América del Sur (Martuscelli-Quintana, 1998).

- Acuerdos que surgen en las reuniones cumbre a las que asisten mandatarios de varios países. Por esta vía México ha conseguido proyectos de cooperación principalmente con la Unión Europea, Estados Unidos y Canadá (Organization of American States, 2006).

El medio por el cual México se integra a los proyectos en colaboración es realmente importante porque es necesario identificar las formas que existen, y tratar de buscar nuevas alternativas que permitan la cooperación internacional. Actualmente esta es una de las formas de hacer ciencia que más se aplica a nivel mundial, sobre todo en los últimos veinte años donde la colaboración científica ha jugado un papel primordial, en la que intervienen distintas especialidades a fin de lograr la sostenibilidad en ciertos campos de investigación, generando ventajas económicas para las naciones pero sin dañar al medio ambiente y a la sociedad (Bettencourta y Kaur, 2011). En este nuevo esquema existe la participación no sólo de las ciencias médicas, sino también sociales y biológicas, particularmente las orientadas al estudio de los recursos del mar y ecología humana, que tienen que ver con los cambios climáticos del planeta y la forma en que perjudican a la población y a las ciudades (Sonnenwald, 2007). Sin duda ésta también es una de las razones por la que se ha incrementado la colaboración en temas de estudio como recursos del mar y medio ambiente (Beltrán-Rodríguez, 2009).

\section{REFERENCIAS}

Aguado-López, E., Rogel-Salazar, R., Garduño-Oropeza, G., Becerril-García, A., Zúñiga-Roca, M.F. y Velázquez-Álvarez, A. (2009), "Patrones de colaboración científica a partir de redes de coautoría", en Convergencia revista de Ciencias Sociales, IA: 225 258.

Alonso-Arroyo, A., Pulgarín, A. y Gil-Leiva, I. (2005), "Estudio cienciométrico de la colaboración científica en la Universidad Politécnica de Valencia, España”, en Information Research, 11 (1): 1-25. 
Aretxaga, I. (2008), “Astronomy. In Science in México 2008: present state and perspectives”, en J.P. Laclette (Ed.). México: Academia Mexicana de Ciencias, pp. 1-39.

Arvanitis, R. (2010), "White paper on strategic indicators for the measurement and impact of international scientific cooperation and collaborations in the Mediterranean region: Report of WP2 of MIRA project”, Beirut: Bondy and Tunis, 62 p. Disponible en: http:// www.cnrs.edu.lb/LORDI\&MIRA-Workshop/White\%20PaperFeb2011.pdf (febrero, 2012).

Atlas de la Ciencia Mexicana (2010), Atlas de la Ciencia Mexicana: Bilingual Edition, México: Conacyt: Complejidad Ciencia y Sociedad, $1 \mathrm{v}$.

Batajelj, V. \& Mrvar, A. (2010), "Pajek Program for Analysis and Visualization of Large Networks: reference manual: list of commands with short explanation version 2.00", Slovenia: University of Ljubjana. 90 p, disponible en línea: http://vlado.fmf.uni-lj.si/ pub/networks/pajek/doc/pajekman.pdf, (agosto, 2011).

Beaver, D. (2001), "Reflections on scientific collaboration (and its study): past, present, and future", en Scientometrics, 52(3), 365377.

Beltrán-Rodríguez, I. (2009), "Evolución de la producción e impacto de la investigación mexicana en ciencias marinas 1984-2004, vista a través de tres sistemas de información SCI, ASFA y PERIODICA", Tesis de Maestría, UNAM, FFyL: CUIB, México, 111 p.

Bettencourta, L.M. and Kaur, J. (2011), "Evolution and structure of sustainability science”, en PNAS, 108 (49): 19540-19545.

Glänzel, W. and Schubert, A. (2001), "Double effort double impact? A critical view of international co-authorship in Chemistry", en Scientometrics, 50: 199-214.

Karlsson, J., Anderbeg, E., Booth, S., Odenrick, P. and Christmansson, M. (2008), "Reaching beyond Disciplines through Collaboration: Academics' Learning in a National Multidisciplinary Research Programme", en Journal of Workplace Learning, 20 (2): 98-113.

Leydesdorff, L. (2006), "The Knowledge-based Economy. Modeled, measured, simulated”, Boca-Ratón, FL: Universal Publishers, 392 p.

Luna-Morales, M.E. y Russell, J, (2009), "El uso de nuevas tecnologías de información y comunicación científica en el área de partículas elementales: el caso de la física mexicana”, México: UNAM, CUIB. 133 p.

Luna-Morales, M.E. y Collazo-Reyes, F. (2005), "Repercusión de un descubrimiento Big Science, de acuerdo con dos modelos de comunicación científica: el caso del quark top", en Revista Española de Documentación Cientifica, 28 (1): 11-21.

(2002), "El síndrome bigscience y su influencia en el proceso de maduración de la física mexicana de partículas elementales", en Revista Española de Documentación Cientifica, 25 (4): 409420 . 
Macrina, F.L. (2005), "Scientific integrity: text and cases in responsible conduct of research", 3ra. ed. Washington, DC: Press American Society for Microbiology Press, 402 p.

Manual de Santiago (2007), "Manual de indicadores de internacionalización de la ciencia y la tecnología”, Chile: RICYT, 66 p.

Martuscelli-Quintana, J. (1998), Promoción y fomento de la investigación en CONACYT/México, Washington, D.C.: Organización Panamericana de la Salud, p. 14

Miranda, J.L., Molina, J.L., Lerner, J., Brandes, U., Ávila, J. and McCarty, C. (2010), "Longitudinal analysis of personal networks. The case of Argentinean migrants in Spain”, en Social Networks, 31 (5): 1-20.

Noguera-Solano, R. Ruiz-Gutiérrez, Y.R. (2000), "El proyecto genoma humano", en Ciencias, 58 (abril-junio): 1-13.

Organization of American States (2006), "Science, Technology, Engineering and Innovation for Development: A vision for the Americans in the Twenty First Century", Washington, D.C.: OEA, 100 p, disponible en: http://www.oest.oas.org/engineering/ingles/documentos/ingles_web.pdf. (Febrero, 2012).

Pillaid, K.G. (2007), "Authorship patterns in physics literature: An informetric study", en Annals of Library and Information Studies, 54: 90-94.

Rivera, A. (1999), "El gran telescopio de Canarias da su paso definitivo al adjudicar la fabricación del espejo principal”, en El País (febrero 02).

Russell, J., Madera-Jaramillo, M.J. y Ainsworth, S. (2009), "El análisis de redes en el estudio de la colaboración científica", en REDES Revista hispana para el análisis de redes sociales, 17 (2):39-47.

Russell, J.M., Madera-Jaramillo, M.J., Hernández-García, Y. and Ainsworth, S. (2008), "Mexican collaboration networks in the international and regional arenas", en: H. Kretschmer \& F. Havemann (Eds.). Fourth International Conference on Webometrics, Informetrics and Scientometrics \& Ninth COLlnET Meeting Humboldt-Universität zu Berlin (Berlin, 2008), pp. 1-10.

Russell, J., Ainsworth, S. y Narvaéz-Berthelemot, N. (2006), "La colaboración científica de la Universidad Nacional Autónoma de México (UNAM) y su política institucional”, en Revista Española de Documentación Cientifica, 29 (1): 56-73.

Russell, J. (1998). "The Increasing Role of International Cooperation in Science and Technology Research in Mexico", en Scientometrics, 34 (1): 45-61.

Sancho, Morillo, F., De Filippo, D., Gómez, I. y Fernández, M.T. (2006), "Indicadores de Colaboración Científica Inter-Centros en los Países de América Latina”, Interciencia, 31 (4): 284-292.

Shin, C.J and Cummings, W.K. (2010), "Multilevel analysis of academic publishing across disciplines: research preference, collaboration, and time on research", en Scientometrics, 85: 581-594. 
Somsen, G. (2008), "A History of Universalism: Conceptions of the Internationality of Science from the Enlightenment to the Cold War", en Minerva, 46: 361-379.

Sonnenwald, D. H. (2007), "Scientific Collaboration", en B. Cronin (Eds.), Annual Review of Information Science and Technology. Nedford, NJ: Information Today, (41): 643-681.

$\infty$ 